\title{
Hemodynamic Adaptations to Regular Exercise in People With Spinal Cord Injury
}

\author{
Young Hee Lee, MD, $\mathrm{PhD}^{1,2}$, Jin Hyeong Lee, $\mathrm{MD}^{1}$, Sung Hoon Kim, MD, $\mathrm{PhD}^{1}$, \\ Dongsoo Yi, $\mathrm{MD}^{1}$, Kyung Joon Oh, $\mathrm{MD}^{1}$, Ji Hyun Kim, $\mathrm{MD}^{1}$, Tae Jun Park, MD ${ }^{1}$, \\ Hanul Kim, $\mathrm{PhD}^{2}$, Jae Seung Chang, $\mathrm{PhD}^{2}$, In Deok Kong, $\mathrm{MD}, \mathrm{PhD}^{2,3}$
}

${ }^{1}$ Department of Rehabilitation Medicine, Yonsei University Wonju College of Medicine, Wonju; ${ }^{2}$ Center for Exercise Medicine, Yonsei University, Wonju; ${ }^{3}$ Department of Physiology, Yonsei University Wonju College of Medicine, Wonju, Korea

\begin{abstract}
Objective To investigate the real-time cardiovascular response to the progressive overload exercise in different levels of spinal cord injury (SCI), and to find out whether regular exercise has effect on these cardiovascular responses.

Methods The study enrolled 8 able-bodied individuals in the control group plus 15 SCI subjects who were divided into two groups by their neurological level of injury: high-level SCI group (T6 or above) and low-level SCI group (T7 or below). Also, subjects were divided into exercise group and non-exercise group by usual exercise habits. We instructed the subjects to perform exercises using arm ergometer according to the protocol and checked plethysmograph for the real time assessment of blood pressure, heart rate, and cardiac output.

Results Six subjects were included in high-level SCI group (3 cervical, 3 thoracic injuries), 9 subjects in low-level SCI group (9 thoracic injuries), and 8 able-bodied individuals in control group. During arm ergometer-graded exercise, mean arterial pressure (MAP) was significantly lower in high-level SCI subjects of non-exercise group, compared with high-level SCI subjects of exercise group. In addition, HR was significantly higher in low-level SCI group compared with control group.

Conclusion There are significant differences in mean arterial pressure of high-level SCI group according to usual exercise habits. We discovered that even in non-athlete high-level SCI, regular exercise can bring cardiac modulation through blood pressure control.
\end{abstract}

Keywords Spinal cord injuries, Ergometer, Exercise test, Aerobic exercise, Cardiovascular physiological phenomena

Received March 21, 2016; Accepted July 14, 2016

Corresponding author: Jin Hyeong Lee

Department of Rehabilitation Medicine, Wonju Severance Christian Hospital, 20 Ilsan-ro, Wonju 26426, Korea. Tel: +82-33-741-1765, Fax: +82-33-7421409, E-mail: eyeini@naver.com

ORCID: Young Hee Lee (http://orcid.org/0000-0001-6861-4250); Jin Hyeong Lee (http://orcid.org/0000-0003-0474-4595); Sung Hoon Kim (http:// orcid.org/0000-0001-6043-7640); Dongsoo Yi (http://orcid.org/0000-0002-5764-0610); Kyung Joon Oh (http://orcid.org/0000-0002-9662-1834); Ji Hyun Kim (http://orcid.org/0000-0001-9061-3350); Tae Jun Park (http://orcid.org/0000-0003-4835-5778); Hanul Kim (http://orcid.org/0000-0002-55901899); Jae Seung Chang (http://orcid.org/0000-0002-4047-1128); In Deok Kong (http://orcid.org/0000-0002-9821-6103).

@ This is an open-access article distributed under the terms of the Creative Commons Attribution Non-Commercial License (http://creativecommons.org/ licenses/by-nc/4.0) which permits unrestricted noncommercial use, distribution, and reproduction in any medium, provided the original work is properly cited. Copyright $\odot 2017$ by Korean Academy of Rehabilitation Medicine 


\section{INTRODUCTION}

During exercise, cardiac output (CO) increases to provide the flow needed to serve the contracting skeletal muscles [1]. However, spinal cord injury (SCI) can lead to an interruption of pathways of the autonomic nervous system (ANS) from its central origins in the brain to the peripheral organ innervations, thus affecting autonomic control of various organs, including those of the cardiovascular system [2-5]. The sympathetic nervous system innervates both the cardiac muscle and smooth muscles of peripheral blood vessels, and is predominant in times of physiological stress and exercise, acting to increase the heart rate (HR) and blood pressure (BP) $[2,5]$. Sympathetic innervation to the heart and majority of blood vessels for upper extremity originates from the upper thoracic segments (T1-T5) [2,6-8]. Injury below T6 tends to spare the control of the heart and most blood vessels, whereas SCI above T6 can interrupt supraspinal sympathetic regulation of the heart, after which spinal circuits become solely responsible for the generation of sympathetic activity below the level of injury $[2,3,6]$.

It is well known that baseline resting BP is inversely correlated with the level of SCI; individuals with high-level SCI have resting systolic BPs of about $15-20 \mathrm{mmHg}$ lower than able-bodied individuals [4-9]. The most commonly described phenomenon in response to exercise in highlevel SCI is poor HR and BP response [10]. Other studies consistently showed that SCI athletes with injuries above T6 have lower maximal HR due to altered sympathetic tone and lower catecholamine release [11-14], and lower maximal oxygen uptake $\left(\mathrm{VO}_{2}\right)[11,12]$ in response to submaximal and maximal exercise in comparison with athletes with lower levels of SCI and athletes with intact ANS. It has also been observed that athletes with cervical SCI have even lower maximal $\mathrm{HR}$ and peak $\mathrm{VO}_{2}$ in response to exercise compared with athletes injured between $\mathrm{Tl}$ and $\mathrm{T} 6$ [13].

In the able-bodied population, higher fitness levels have been related to improved autonomic cardiovascular control, and conversely, physical inactivity has been associated with reduced autonomic cardiovascular tone $[15,16]$. Athletes with SCI have also demonstrated some ability to improve cardiovascular modulation through exercise $[17,18]$, although not to the extent of athletes with intact ANS $[19,20]$.
Although a number of studies have examined cardiovascular control in people with SCI during exercise, there is limited information on the effect of regular exercise habits. While previous studies were carried out among athletes $[13-15,19,20]$, the subjects that we were particularly interested in were non-athlete-SCI individuals who conducted exercise. There have been no studies on the cardiovascular change in real-time hemodynamic adaptations according to habitual exercise in SCI.

Currently, as the life expectancy and survival rate of SCI individuals improve, the need for higher quality of life as well as participation in physical activities and recreational sports is also increasing. However, there is risk of cardiovascular complications if these physical activities and recreational sports are not done in proper exercise intensity.

This study aims to measure real-time cardiovascular response in SCI and in healthy individuals for comparative analysis between varying neurological levels of injury and exercise habits. This is intended to provide instructions on proper exercise intensity and confirm the effect of regular exercise habit on cardiovascular function in SCI.

\section{MATERIALS AND METHODS}

\section{Subjects}

Fifteen subjects with SCI of $>2$ years duration and 8 normal individuals were included in the study as treatment and control groups, respectively. All subjects were over 20 years old. The exclusion criteria included SCI with arrhythmia, dyspnea, orthopedic problems, skin breakdown, and pressure ulcers; in addition, subjects who could not sustain upright position on the wheelchair for at least an hour were excluded. The subjects were interviewed for their demographic information including age, sex, weight, height, body mass index, time since SCI, type of exercise, and health-related physical fitness.

The SCI subjects were divided into two groups by their neurological level of injury based on the International Standards for Neurological Classification of Spinal Cord Injury. The lesions of SCI were stable in all patients. The high-level SCI group consisted of those with neurological level of injury at T6 or above; whereas, the low-level SCI group consisted of those with neurological level of injury at $\mathrm{T} 7$ or below. 
Also, subjects were divided into the exercise group and non-exercise group. The exercise group regularly participated in at least 3 sessions of one or more sport activities per week, or in exercise lasting a minimum of $150 \mathrm{~min}$ utes per week. The exercise habits were measured by Godin Leisure-Time Exercise Questionnaire [21].

\section{Cardiovascular fitness measurements}

Plethysmography was used for non-invasive beat-tobeat blood pressure monitoring, providing hemodynamic parameters such as stroke volume, total peripheral resistance and cardiac output, as well as pulse rate and baroreflex sensitivity analysis.

The examiner provided instructions for the plethysmograph (Finometer PRO; Finapres Medical Systems, Amsterdam, The Netherlands) and carried out beat-tobeat assessment of $\mathrm{BP}, \mathrm{HR}$, and $\mathrm{CO}$, respectively. A plethysmograph was attached to the great toe to assess cardiovascular response during the arm ergometer exercise. The electrocardiograph (ECG) was recorded with ECG monitoring system (Q-Stress v3.5; Quinton, Bothell, WA, USA).

\section{Experimental design}

All subjects performed a graded exercise protocol using arm ergometer (Angio 917904; Lode BV, Groningen, The Netherlands) and went through the procedures in their personal wheelchairs or adjusted chair. Duration of exercise stage, amount of workload, and workload increments in each stage were set according to the generic exercise testing protocol [22].

The exercise began with a 2-minute warm-up and proceeded stepwise with increasing intensity by 2 -minute intervals until stage 5 . Initial workload was $30 \mathrm{~W}$ and the speed was maintained at 60 revolutions per minute (rpm). The loading was increased by $15 \mathrm{~W}$ for each exercise stage and the protocol was carried out until stage 5 (Table 1 ).

The arm-ergometer exercise was discontinued when the cycling rate dropped below $30 \mathrm{rpm}$ or when the subject experienced maximal exertion or wanted to stop the protocol.

\section{Data analysis}

All data were analyzed using SPSS ver. 19.0 (IBM, Armonk, NY, USA). Comparisons between the two groups were performed using Mann-Whitney test, while Krus-
Table 1. Graded exercise protocol using arm ergometer

\begin{tabular}{lccc}
\hline \multicolumn{1}{c}{ Stage } & $\begin{array}{c}\text { Load } \\
\text { (W) }\end{array}$ & $\begin{array}{c}\text { Duration } \\
(\mathbf{m i n})\end{array}$ & METs \\
\hline 0 (warm up) & 0 & $2: 00$ & 1.9 \\
1 & 30 & $2: 00$ & 4.6 \\
2 & 45 & $2: 00$ & 7.0 \\
3 & 60 & $2: 00$ & 10.1 \\
4 & 75 & $2: 00$ & 12.8 \\
5 & 90 & $2: 00$ & 14.8 \\
\hline
\end{tabular}

MET, metabolic equivalents of task.

kal-Wallis test and Mann-Whitney test were used to compare the data obtained from all three groups. Repeated measures ANOVA was used to examine mean differences in assessment of cardiovascular response during the exercise protocol. Statistical significance was determined at $\mathrm{p}<0.05$.

\section{RESULTS}

\section{Subject characteristics}

Of the 8 subjects in high-level SCI group, two dropped out during stage 2 of the graded exercise protocol due to lack of upper extremity power, while the other 6 subjects completed the graded exercise protocol. All nine subjects in the low-level SCI group completed the graded exercise protocol.

Age, body mass index, and Godin leisure-time exercise score showed no significant differences between highlevel SCI group, low-level SCI group and control group. The neurological level of injury of SCI subjects involved 3 cervical, 3 thoracic in high-level SCI group, and 9 thoracic in low-level SCI group (Table 2).

Among high-level SCI group, 3 subjects performed regular exercises, and 5 of low-level SCI group and 4 subjects of control group also went through regular exercises.

The age of the subjects ranged from 23 to 60 years, with the average of $37.78 \pm 11.23$ years. Among 23 subjects, 12 were under 39 years of age, and 11 were 40 years old or older.

The change in cardiovascular response during arm ergometer graded exercise

The mean arterial pressure (MAP) of high-level SCI group, low-level SCI group, and control group at warm 
Table 2. Demographic and clinical characteristics

\begin{tabular}{|c|c|c|c|c|}
\hline & $\begin{array}{l}\text { High-level SCI group } \\
(\mathrm{n}=6)\end{array}$ & $\begin{array}{c}\text { Low-level SCI group } \\
(\mathrm{n}=9)\end{array}$ & $\begin{array}{c}\text { Control group } \\
(n=8)\end{array}$ & p-value \\
\hline Gender (male:female) & $5: 1$ & $8: 1$ & $8: 0$ & 0.535 \\
\hline Age (yr) & $40.50 \pm 9.23$ & $41.78 \pm 13.04$ & $31.25 \pm 8.12$ & 0.230 \\
\hline $\operatorname{BMI}\left(\mathrm{kg} / \mathrm{m}^{2}\right)$ & $23.27 \pm 2.98$ & $21.93 \pm 2.746$ & $24.49 \pm 2.38$ & 0.264 \\
\hline \multicolumn{5}{|l|}{ Injury level } \\
\hline Cervical & 3 & 0 & - & - \\
\hline Thoracic & 3 & 9 & - & - \\
\hline Lumbar & 0 & 0 & - & - \\
\hline \multicolumn{5}{|l|}{ AIS } \\
\hline A & 3 & 8 & - & \\
\hline $\mathrm{B}$ & 2 & 0 & - & \\
\hline $\mathrm{C}$ & 1 & 1 & - & \\
\hline $\mathrm{D}$ & 0 & 0 & & \\
\hline Time since injury (yr) & $12.33 \pm 5.68$ & $12.33 \pm 6.18$ & - & 0.864 \\
\hline Regular exercise (yes:no) & $3: 3$ & $5: 4$ & $4: 4$ & 0.744 \\
\hline Weekly leisure activity score & $48.16 \pm 29.28$ & $68.67 \pm 22.82$ & $43.50 \pm 30.43$ & 0.859 \\
\hline
\end{tabular}

Values are presented as number or mean \pm standard deviation.

SCI, spinal cord injury; high-level SCI group, neurological level of injury T6 or above; low-level SCI group, neurological level of injury T7 or below; AIS, American Spinal Injury Association impairment scale; BMI, body mass index.

Table 3. Effect of injury level on cardiovascular parameters

\begin{tabular}{llcccccc}
\hline & & $\begin{array}{c}\text { Stage 0 } \\
\text { (warm up) }\end{array}$ & Stage 1 & Stage 2 & Stage 3 & Stage 4 & Stage 5 \\
\hline MAP (mmHg) & High-level SCI & $83.3 \pm 21.4$ & $83.8 \pm 24.1$ & $86.5 \pm 25.4$ & $86.5 \pm 26.9$ & $86.5 \pm 26.9$ & $96.0 \pm 28.7$ \\
& Low-level SCI & $83.6 \pm 20.3$ & $91.7 \pm 21.9$ & $100.1 \pm 19.1$ & $107.7 \pm 19.7$ & $114.6 \pm 20.4$ & $122.3 \pm 24.7$ \\
& Control & $74.4 \pm 11.7$ & $86.1 \pm 14.8$ & $88.2 \pm 18.4$ & $98.7 \pm 20.8$ & $112.2 \pm 17.9$ & $114.9 \pm 25.1$ \\
\hline \multirow{2}{*}{ HR (bpm) } & High-level SCI & $85.6 \pm 13.7$ & $91.2 \pm 16.8$ & $98.9 \pm 19.5$ & $108.9 \pm 19.8$ & $127.3 \pm 19.9$ & $141.9 \pm 24.8$ \\
& Low-level SCI & $90.7 \pm 8.2$ & $97.1 \pm 8.0^{*}$ & $106.3 \pm 9.3^{*}$ & $119.6 \pm 9.4^{*}$ & $134.7 \pm 11.3^{*}$ & $149.6 \pm 11.3^{*}$ \\
& Control & $77.5 \pm 12.6$ & $77.0 \pm 12.1$ & $82.6 \pm 15.4$ & $94.9 \pm 14.0$ & $107.3 \pm 16.4$ & $122.5 \pm 19.4$ \\
\hline CO (L/min) & High-level SCI & $10.0 \pm 3.6$ & $10.5 \pm 3.9$ & $11.4 \pm 4.7$ & $12.8 \pm 5.4$ & $14.9 \pm 5.9$ & $17.4 \pm 6.4$ \\
& Low-level SCI & $8.6 \pm 4.2$ & $9.5 \pm 4.0$ & $10.7 \pm 4.41$ & $12.1 \pm 4.7$ & $13.7 \pm 5.5$ & $14.5 \pm 6.2$ \\
& Control & $9.2 \pm 2.6$ & $9.7 \pm 2.4$ & $10.7 \pm 2.9$ & $12.5 \pm 4.0$ & $13.7 \pm 2.3$ & $14.5 \pm 3.0$ \\
\hline
\end{tabular}

Values are presented as mean \pm standard deviation.

MAP, mean arterial pressure; HR, heart rate; CO, cardiac output; SCI, spinal cord injury.

${ }^{*} p<0.05$, significant difference of HR between low-level SCI and control groups. No significant difference among the MAP and CO in high-level SCI and low-level SCI and control groups.

up stage was $83.3 \pm 21.4 \mathrm{mmHg}, 83.6 \pm 20.3 \mathrm{mmHg}$, and $74.4 \pm 11.7 \mathrm{mmHg}$, respectively, and increased by each stage of exercise (Table 3). The slope of the MAP versus time curves (MAP inclination) was lower in high-level SCI group, as compared to low-level SCI group $(0.005 \pm 0.026$ $\mathrm{mmHg} / \mathrm{s}$ vs. $0.033 \pm 0.010 \mathrm{mmHg} / \mathrm{s} ; \mathrm{p}<0.05)$ and the control group $(0.005 \pm 0.026 \mathrm{mmHg} / \mathrm{s}$ vs. $0.053 \pm 0.017 \mathrm{mmHg} / \mathrm{s}$; $\mathrm{p}<0.001$ ) (Fig. 1A). During stages 0 to 4 , MAP was significantly lower in high-level SCI subjects of non-exercise group, as compared with high-level SCI subjects of exercise group (stage $0,99.80 \pm 8.69$ vs. $66.74 \pm 15.82 \mathrm{mmHg}$; stage $1,104.04 \pm 6.11$ vs. $63.65 \pm 13.79 \mathrm{mmHg}$; stage 2 , $103.37 \pm 8.37$ vs. $61.14 \pm 11.54 \mathrm{mmHg}$; stage $3,103.59 \pm 10.92$ vs. $60.02 \pm 17.70 \mathrm{mmHg}$; stage $4,104.22 \pm 10.92$ vs. $60.02 \pm$ 

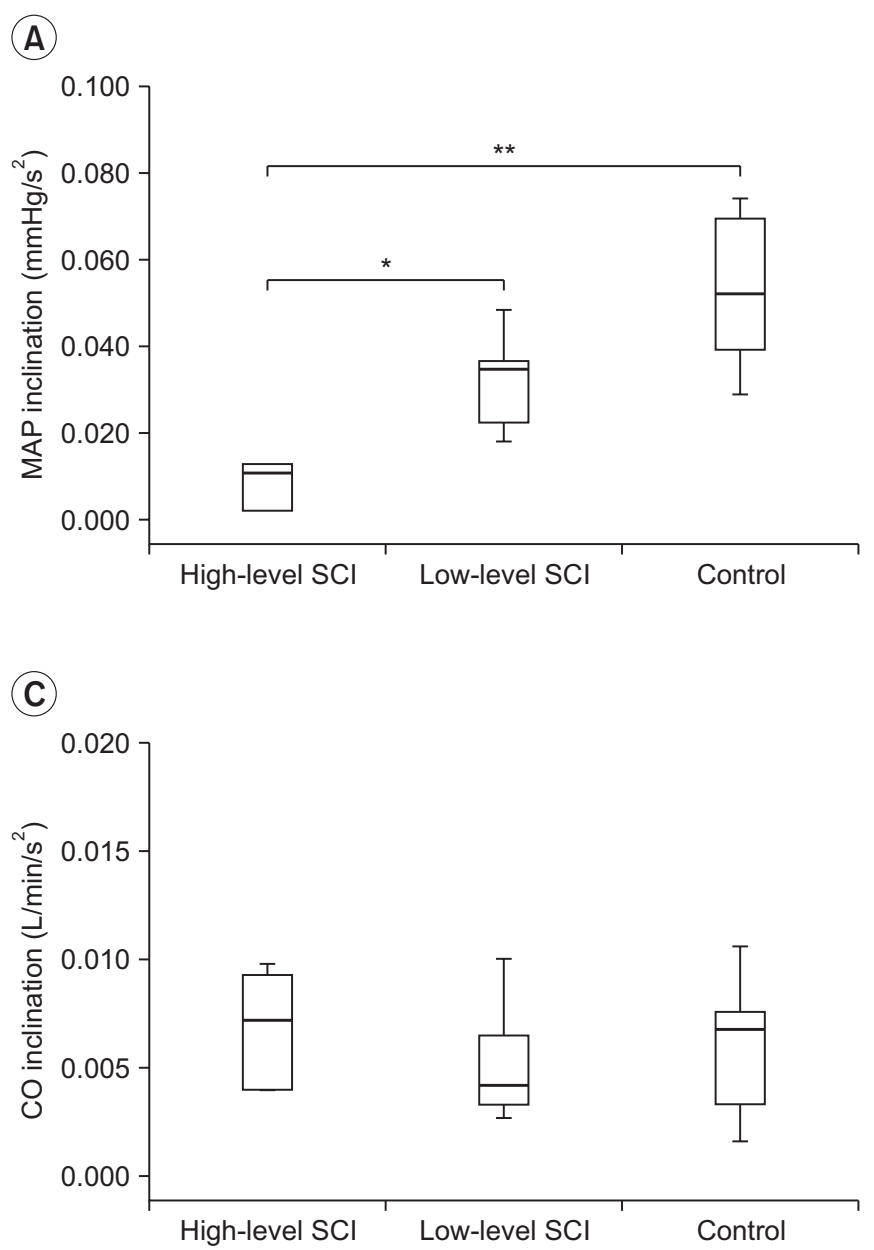

17.70 mmHg; p<0.05) (Fig. 2A).

During stages 1 to 5, HR was higher in low-level SCI group, as compared with control group (stage 1, 97.1 \pm 8.0 vs. $77.0 \pm 12.1 \mathrm{bpm}$; stage $2,106.3 \pm 9.3$ vs. $82.6 \pm 15.4 \mathrm{bpm}$; stage $3,119.6 \pm 9.42$ vs. $94.9 \pm 14.0 \mathrm{bpm}$; stage $4,134.7 \pm 11.3$ vs. $107.3 \pm 16.4 \mathrm{bpm}$; stage $5,149.6 \pm 11.3$ vs. $122.5 \pm 19.4$ bpm; $\mathrm{p}<0.05)$ (Table 3$)$. The slopes of the HR versus time curves (HR inclination) showed no significant differences in the three groups (Fig. 1B).

During arm ergometer graded exercise, cardiac output increased by each stage of exercise in the three groups (Table 3). However, CO showed no additional increase from stage 4 to stage 5 in low-level SCI group (stage 4 , $13.7 \pm 5.5 \mathrm{~L} / \mathrm{min}$; stage $5,14.5 \pm 6.2 \mathrm{~L} / \mathrm{min}$ ) and control group (stage $4,13.7 \pm 2.3 \mathrm{~L} / \mathrm{min}$; stage $5,14.5 \pm 3.0 \mathrm{~L} / \mathrm{min}$ ), while additional increase was observed in high-level SCI group (stage 4, 14.9 $\pm 5.9 \mathrm{~L} / \mathrm{min}$; stage $5,17.4 \pm 6.4 \mathrm{~L} / \mathrm{min}$ ). The slopes of the $\mathrm{CO}$ versus time curves (CO inclination) were not significantly different in the three groups (Fig.

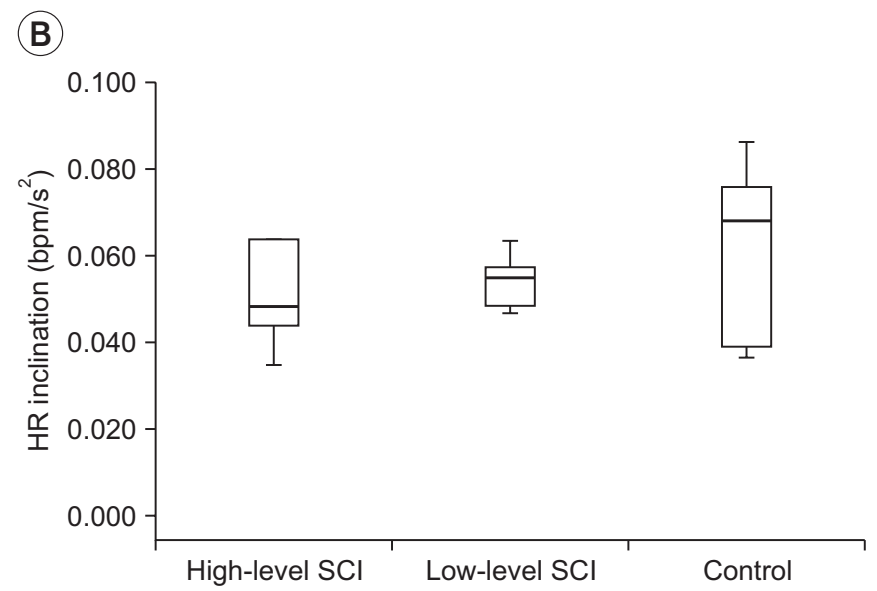

Fig. 1. The inclination of MAP (A), HR (B) and CO (C) by neurologic levels. Based on neurologic injury level, highlevel SCI show significantly lower MAP inclination than low-level SCI and control. ${ }^{*} \mathrm{p}<0.05$, significant difference between high-level SCI and low-level SCI. ${ }^{* *}$ p $<0.001$, between high-level SCI and control. No significant difference was found in HR inclination (B) and $\mathrm{CO}$ inclination (C). MAP, mean arterial pressure; HR, heart rate; $\mathrm{CO}$, cardiac output; SCI, spinal cord injury.

1C). During arm ergometer graded exercise, $\mathrm{CO}$ was lower in non-exercise group than exercise group (Fig. 2C).

\section{DISCUSSION}

Exercise is important to individuals with SCI since it is known to play a major role in preventing complications and decreased quality of life that can result from lack of physical activities. Gass et al. [23] reported that subjects with high level of spinal lesion can exhibit improved cardiovascular function through regular aerobic exercises.

However, due to different environments, individuals with spinal cord injured are expected to conduct different exercise regimens. This study used an arm ergometer to directly compare hemodynamic adaptations of subjects from high-level SCI group, low-level SCI group and control group by their history of regular exercise.

Mathias and Frankel [24] reported an inverse relationship between the level of SCI and resting blood pressure. 

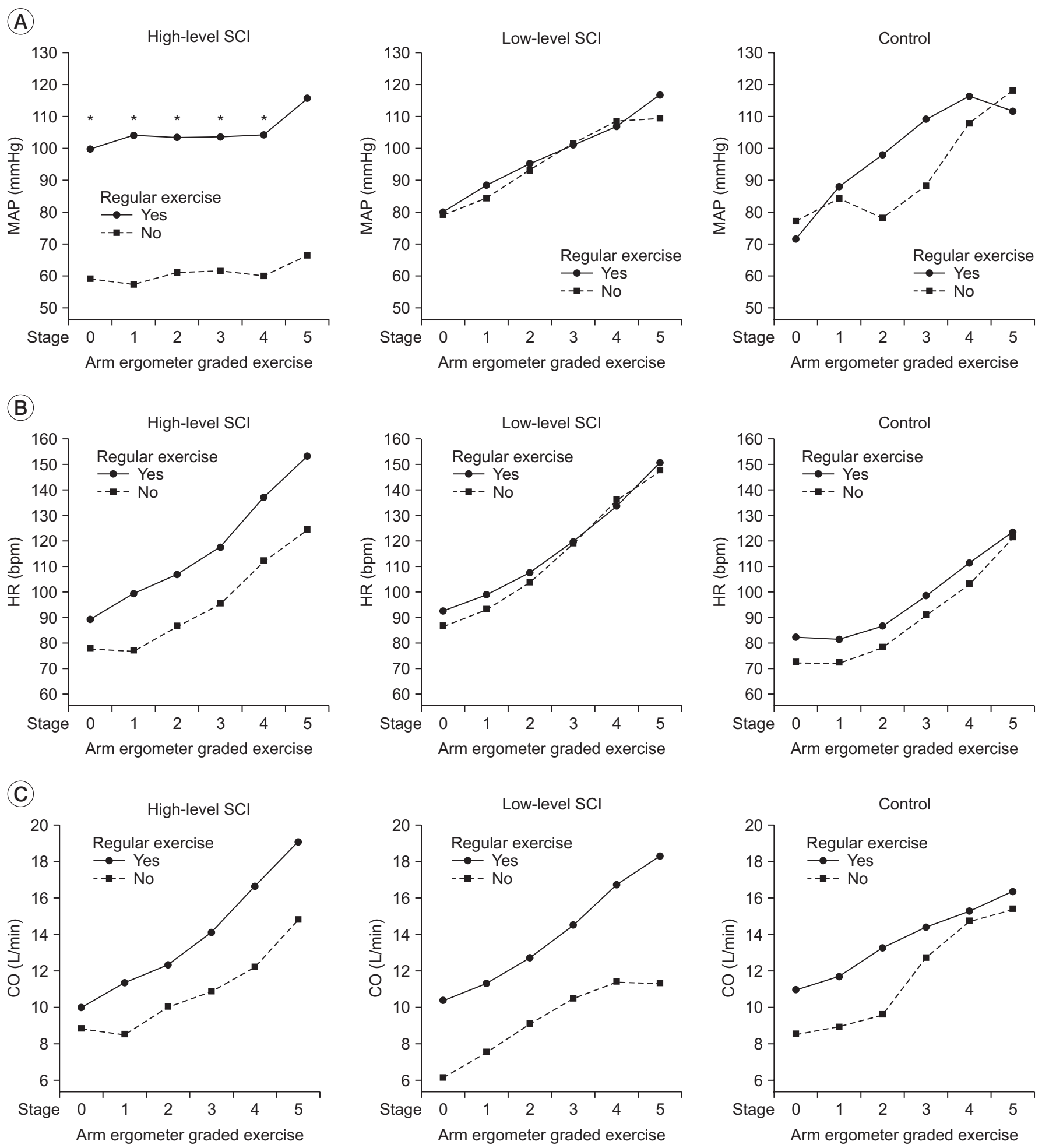

Fig. 2. The MAP (A), HR (B) and CO (C) in response to arm ergometer graded exercise in high-level SCI, low-level SCI, and control based on exercise habit. The MAP showed significant difference between high-level SCI with regular exercise and high-level SCI without regular exercise during stage $0,1,2,3,4(\mathrm{p}<0.05) .{ }^{*} \mathrm{p}<0.05$ between high-level SCI with regular exercise and high-level SCI without regular exercise during stage $0,1,2,3$, 4 . No significant difference was found in HR (B) and CO (C) in high-level SCI, low-level SCI, and control by exercise habit. MAP, mean arterial pressure; HR, heart rate; $\mathrm{CO}$, cardiac output; SCI, spinal cord injury. 
However, the results showed no significant differences of MAP between the three groups at warm up stage; and the mean value of MAP in control group was slightly lower than that of MAP in high-level SCI group and low-level SCI group. Since subjects in stage 0 (warm up phase) performed arm ergometer in seating position without loading, resting BP could affected differently as compared with other studies; which could have caused higher MAP in low-level SCI group and high-level SCI group than control group. Additional studies with more subjects are needed for the cardiac response in warm up phase in various injury levels of SCI.

Claydon et al. [25] studied cardiovascular response with continuous BP using beat-to-beat BP monitoring device and electrocardiographic monitoring in chronic cervical $(n=8)$ and upper thoracic SCI $(n=5)$; the resting MAP was lower in subjects with cervical SCI than thoracic SCI (64.1 $\pm 6.6 \mathrm{mmHg}$ vs. $79.4 \pm 8.7 \mathrm{mmHg}$ ). However, they enrolled subjects who were apparently healthy, free from pain, and without any known cardiovascular disease or pressure ulcers, while exercise habits were not mentioned. In this study, the MAP of non-exercise group of high SCI level in stage 0 was $66.74 \pm 15.82 \mathrm{mmHg}$. The low BP in high-level SCI could have resulted from cardiovascular assessment using plethysmograph contacted to the 1st toe, since in high-level SCI, blood volume is sequestered in the leg veins resulting in reduced mean systemic filling pressure at the heart [26].

In this study, during arm ergometer graded exercise, MAP was significantly lower in non-exercise group of high-level SCI compared with exercise group of high-level SCI. This is explained by the poor HR and BP response to exercise in high-level SCI individuals [10]. In highlevel SCI individuals, non-athletes may be secondary to impairments in sympathetic function [27], and impaired sympathetic innervation could influence in cardiac contractility and HR. Meanwhile, HR was higher in low-level SCI compared with the control. Schmid et al. [28] studied catecholamines, HR, and oxygen uptake during exercise in persons with SCI and showed that paraplegics with a lesion level below $\mathrm{T} 5$ had higher resting and maximal HR than higher level paraplegics and abled bodied subjects. In their study, higher maximal cardiovascular and metabolic performance capacity was due to the intact noradrenergic sympathetic innervation of the heart and muscles of the upper body by efferent sympathetic fibers.
During exercise, $\mathrm{CO}$ increases to provide the flow needed to serve the contracting skeletal muscles [29]. As exercise progresses, all groups show increase in $\mathrm{CO}$, reflecting the increased blood supply requirement as exercise progresses. In low-level SCI group and control group, the slopes of the CO decreased with progress in exercise, whereas high-level SCI showed increase in slopes of CO as exercise progressed. HR and stroke volume are the major factors influencing CO. In this study, high-level SCI group showed significantly lower MAP inclination, but similar HR increases; therefore, SCI group maintained $\mathrm{CO}$ at the same workload by HR, rather than stroke volume. In subjects with tetraplegia who showed a decrease in stroke volume, $\mathrm{CO}$ was maintained by an increase in the HR during exercise [30].

Autonomic dysreflexia symptoms (such as extreme hypertension, pounding headache and slow $\mathrm{HR}$, and upper body flushing) were absent during the exercise protocol in SCI subjects. The history of autonomic dysreflexia or orthostatic hypotension in SCI subjects was not considered in this study, hence, we were unable to discover the association between cardiovascular responses and clinical problems in cardiovascular control.

The subjects of this study included few stable SCI individuals, of mixed gender and broad age groups. Other factors, such as age, sex, pulmonary function, history of autonomic dysreflexia or orthostatic hypotension in SCI subjects that may have effect on cardiac measurement were not controlled. Also, the evaluation on the regular exercise level was through Godin Leisure-Time Exercise Questionnaire; therefore, it was difficult to compare the effects based on different exercise types. Since progressive stress exercise was increased arithmetically and not based on each subject's motor power, the stress at each stage could have differed among subjects. Although the plethysmograph should be applied on the finger or brachial area, contacting plethysmograph to the 1st toe was required to measure the BP constantly during arm-ergometer exercise. It is possible that differences might occur when measured in 1st toe. The subjects had varying levels of experience with exercising with arm ergometer. Only 1 set of exercise was assigned per person, contributing to lack of reliability. More detailed assessment of cardiovascular response could have been possible if the post-exercise results were observed after the stress exercise as well as after the warm-up phase. 
This was the first study on real-time cardiovascular response of high-level SCI group, low-level SCI group, and control group during progressive stress exercise. The results showed that even in non-athlete SCI, regular exercise habits can mediate cardiac modulation through BP control in high-level SCI. Low MAP inclination in highlevel SCI group indicated that cardiovascular response to graded exercise was difficult in high-level SCI. Moreover, the exercise group of high-level SCI group had higher MAP than the non-exercise group, suggesting that regular exercise would help cardiovascular modulation by increasing BP. Moreover, HR inclination showed no significant between-group difference.

Additional studies with more subjects and prospective design are needed to assess the effect of regular exercise on the cardiac response in high-level SCI. Measurements of MAP and HR in resting phase to maximal workload are necessary for the mechanisms involved in differential exercise adaptation in the three groups.

This study suggested differences in MAP of high-level spinal cord injury individuals based on exercise habits. Thus, regular exercise can bring about cardiac modulation through blood pressure control in high SCI.

\section{CONFLICT OF INTEREST}

No potential conflict of interest relevant to this article was reported.

\section{ACKNOWLEDGMENTS}

This work was supported by a research grant from Yonsei University Wonju College of Medicine (YUWCM2012-23).

\section{REFERENCES}

1. Clausen JP. Circulatory adjustments to dynamic exercise and effect of physical training in normal subjects and in patients with coronary artery disease. Prog Cardiovasc Dis 1976;18:459-95.

2. Garstang SV, Miller-Smith SA. Autonomic nervous system dysfunction after spinal cord injury. Phys Med Rehabil Clin N Am 2007;18:275-96.

3. Gondim FA, Lopes AC Jr, Oliveira GR, Rodrigues CL, Leal PR, Santos AA, et al. Cardiovascular control after spinal cord injury. Curr Vasc Pharmacol 2004;2:71-9.

4. Krassioukov A, Claydon VE. The clinical problems in cardiovascular control following spinal cord injury: an overview. Prog Brain Res 2006;152:223-9.

5. Bravo G, Guízar-Sahagun G, Ibarra A, Centurion D, Villalon CM. Cardiovascular alterations after spinal cord injury: an overview. Curr Med Chem Cardiovasc Hematol Agents 2004;2:133-48.

6. Krassioukov A. Which pathways must be spared in the injured human spinal cord to retain cardiovascular control? Prog Brain Res 2006;152:39-47.

7. Krassioukov AV, Bunge RP, Pucket WR, Bygrave MA. The changes in human spinal sympathetic preganglionic neurons after spinal cord injury. Spinal Cord 1999;37:6-13.

8. Krassioukov AV, Weaver LC. Reflex and morphological changes in spinal preganglionic neurons after cord injury in rats. Clin Exp Hypertens 1995;17:361-73.

9. Claydon VE, Krassioukov AV. Orthostatic hypotension and autonomic pathways after spinal cord injury. J Neurotrauma 2006;23:1713-25.

10. Claydon VE, Hol AT, Eng JJ, Krassioukov AV. Cardiovascular responses and postexercise hypotension after arm cycling exercise in subjects with spinal cord injury. Arch Phys Med Rehabil 2006;87:1106-14.

11. Hopman MT, Oeseburg B, Binkhorst RA. Cardiovascular responses in persons with paraplegia to prolonged arm exercise and thermal stress. Med Sci Sports Exerc 1993;25:577-83.

12. Rimaud D, Calmels P, Roche F, Mongold JJ, Trudeau F, Devillard X. Effects of graduated compression stockings on cardiovascular and metabolic responses to exercise and exercise recovery in persons with spinal cord injury. Arch Phys Med Rehabil 2007;88:703-9.

13. Campbell IG, Williams C, Lakomy HK. Physiological and metabolic responses of wheelchair athletes in different racing classes to prolonged exercise. J Sports Sci 2004;22:449-56.

14. Barfield JP, Malone LA, Collins JM, Ruble SB. Disability type influences heart rate response during power wheelchair sport. Med Sci Sports Exerc 2005;37:71823.

15. Dixon EM, Kamath MV, McCartney N, Fallen EL. Neural regulation of heart rate variability in endurance athletes and sedentary controls. Cardiovasc Res 1992;26:713-9. 
16. Yamamoto K, Miyachi M, Saitoh T, Yoshioka A, Onodera $\mathrm{S}$. Effects of endurance training on resting and post-exercise cardiac autonomic control. Med Sci Sports Exerc 2001;33:1496-502.

17. Otsuka Y, Shima N, Moritani T, Okuda K, Yabe K. Orthostatic influence on heart rate and blood pressure variability in trained persons with tetraplegia. Eur J Appl Physiol 2008;104:75-8.

18. Wecht JM, Marsico R, Weir JP, Spungen AM, Bauman WA, De Meersman RE. Autonomic recovery from peak arm exercise in fit and unfit individuals with paraplegia. Med Sci Sports Exerc 2006;38:1223-8.

19. Schmid A, Huonker M, Stober P, Barturen JM, Schmidt-Trucksass A, Durr H, et al. Physical performance and cardiovascular and metabolic adaptation of elite female wheelchair basketball players in wheelchair ergometry and in competition. Am J Phys Med Rehabil 1998;77:527-33.

20. Asayama K, Nakamura Y, Ogata H, Hatada K, Okuma $\mathrm{H}$, Deguchi Y. Physical fitness of paraplegics in full wheelchair marathon racing. Paraplegia 1985;23:27787.

21. Godin G, Shephard RJ. A simple method to assess exercise behavior in the community. Can J Appl Sport Sci 1985;10:141-6.

22. Gappmaier E. The Submaximal Clinical Exercise Tolerance Test (SXTT) to establish safe exercise prescription parameters for patients with chronic disease and disability. Cardiopulm Phys Ther J 2012;23:19-29.

23. Gass GC, Watson J, Camp EM, Court HJ, McPherson LM, Redhead P. The effects of physical training on high level spinal lesion patients. Scand J Rehabil Med
1980;12:61-5.

24. Mathias CJ, Frankel HL. Autonomic disturbances in spinal cord lesions. In: Mathias CJ, Bannister R, editors. Autonomic failure: a textbook of clinical disorders of the autonomic nervous system. 4th ed. Oxford: Oxford University Press; 2002. p. 494-513.

25. Claydon VE, Elliott SL, Sheel AW, Krassioukov A. Cardiovascular responses to vibrostimulation for sperm retrieval in men with spinal cord injury. J Spinal Cord Med 2006;29:207-16.

26. Teasell RW, Arnold JM, Krassioukov A, Delaney GA. Cardiovascular consequences of loss of supraspinal control of the sympathetic nervous system after spinal cord injury. Arch Phys Med Rehabil 2000;81:506-16.

27. Currie KD, West CR, Hubli M, Gee CM, Krassioukov AV. Peak heart rates and sympathetic function in tetraplegic nonathletes and athletes. Med Sci Sports Exerc 2015;47:1259-64.

28. Schmid A, Huonker M, Barturen JM, Stahl F, SchmidtTrucksass A, Konig D, et al. Catecholamines, heart rate, and oxygen uptake during exercise in persons with spinal cord injury. J Appl Physiol (1985) 1998;85: 635-41.

29. Dela F, Mohr T, Jensen CM, Haahr HL, Secher NH, Biering-Sorensen F, et al. Cardiovascular control during exercise: insights from spinal cord-injured humans. Circulation 2003;107:2127-33.

30. Hostettler S, Leuthold L, Brechbuhl J, Mueller G, Illi SK, Spengler CM. Maximal cardiac output during arm exercise in the sitting position after cervical spinal cord injury. J Rehabil Med 2012;44:131-6. 